Minimal-invasiv aber effektiv!

\section{Mikrowelle entfernt Lungenmetastasen}

> Ein neues minimal-invasives Verfahren weckt neue Hoffnung für Patienten mit nicht resezierbaren Lungenmetastasen. Es handelt sich um die Mikrowellenablation, ein Verfahren, bei dem man unter lokaler Betäubung, mittels einer Sonde, Mikrowellen direkt auf das Tumorgewebe lenkt. Der Eingriff wird mithilfe von CT-Echtzeitbildern gesteuert und die Bestrahlung dauert im Durchschnitt zwischen fünf und zehn Minuten.

Die Wirksamkeit des Verfahrens wurde in einer Studie der Uniklinik Frankfurt unter der Leitung von Prof. Thomas Vogl bewiesen. Von 130 Tumo-

\section{Asthma als Kind, ADHS als Teenie?}

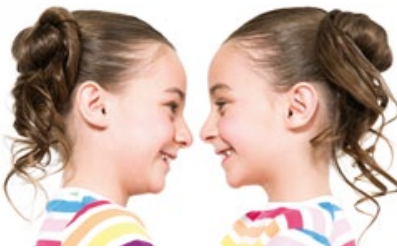

> Dass es einen Zusammenhang zwischen Asthma in der Kindheit und einer Aufmerksamkeitsdefizit/ Hyperaktivitätsstörung (ADHS) in derPubertät gibt, konnten Wissenschaftler in einer Untersuchung mit 906 Zwillingspaaren feststellen. Die Daten erhielten sie mittels Fragebögen und das Fazit war folgendes: Asthma im Alter von acht bzw. neun Jahren geht mit einem zweifach erhöhten Risiko für ADHS im Alter von 13 bzw. 14 Jahren einher. Allergy 2011, 66:1224 ren, bei 80 Probanden, konnten 95 $(73,1 \%)$ dank der neuen Therapie vollständig entfernt werden. Außerdem lag das Ein-Jahres-Überleben bei 91,3\% und das Zwei-Jahres-Überleben bei 75\%.

Die Ärzte stellten fest, dass sowohl die Größe als auch die Lage des Tumors den Therapieerfolg beeinflussen: je kleiner $(<3 \mathrm{~cm})$ und peripherer die Metastasen, desto größer die Erfolgschancen. „Die Mikrowellenablation kann sicher und präzise für alle Arten von Lungenmetastasen eingesetzt werden“, so die Schlussfolgerung der Autoren der Studie.

(bp)

Radiology 2011, 261:643

\section{Klimawandel: Gefahr für COPD-Patienten}

> Nicht nur die Ökosysteme, sondern auch die Gesundheit der Menschen ist vom Klimawandel bedroht. Und besonders gefährdet sind Patienten mit COPD, betonte der Lungenexperte Prof. Christian Witt von der Berliner Charité. Bei den Hitzewellen in den vergangenen Jahren, insbesondere im Sommer 2003, seien nämlich die meisten Menschen nicht an Herzinfarkt oder Kreislaufkollaps gestorben, sondern weil die Lunge versagte. Besonders gefährlich für Lungen und Atemwege sind die Ozonbelastung und die partikelförmigen Schadstoffe. Ärzte Zeitung vom 18.11 und vom 1.12.2011

\title{
Nase voll? Das muss nicht sein!
}

Zufriedenheit hält einem sogar den Schnupfen vom Leib - das meinte zumindest Friedrich Nietzsche. Wenn da was Wahres dran ist, schaut's im Januar nicht allzu rosig aus mit der deutschen Gemütslage. Da wird geschnieft, geprustet und gerotzt was das Zeug hält. Aber ob es nun an Unzufriedenheit oder Wetterlage liegt, hat's einen einmal erwischt, will man die Nasenplage so schnell wie möglich wieder los werden. Was bei akuter Rhinosinusitis wirklich hilft, lesen Sie evidenzgeprüft in unserem Schwerpunkt. Doch nicht nur Nase und Nebenhöhlen, auch der Husten hat im Nasskalten Hochkonjunktur. Wir präsentieren Ihnen im Schwerpunkt einen besonderen Fall, bei dem nicht Viren, sondern der Darm zu trockenen Hustenattacken einer Patientin geführt hatte. Des Rätsels Lösung sowie mehr zu Allergieprävention, Asthmatherapie, COPD-Behandlung und Pulmonaler Hypertonie lesen Sie in unserem aktuellen Schwerpunkt "Atemwege". Eine interessante Lektüre wünscht

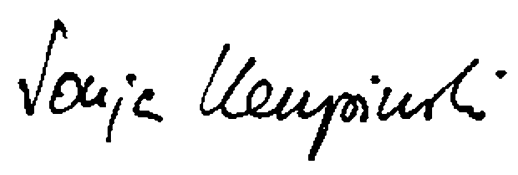

Setzen, 5! Deutsche wissen wenig in puncto Atemwegserkrankung

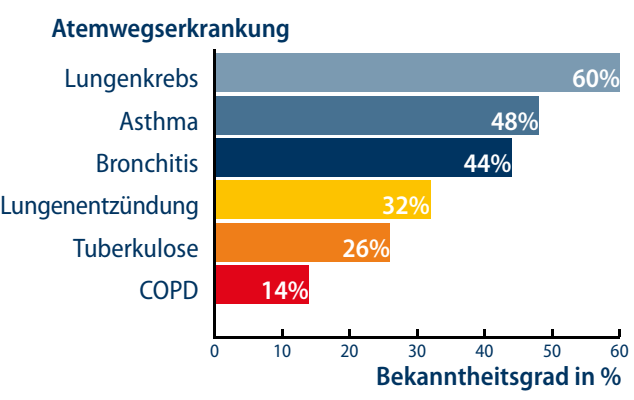

Obwohl es in Deutschland jährlich ca. 4 Mio. Todesfälle gibt, wissen nur 14\% aller Deutschen, was „COPD“ bedeutet. Weitaus bekanntere Lungenerkrankungen sind hingegen Lungenkrebs, Asthma und Bronchitis.

Bundesministerium für Bildung und Forschung, Forsa-Umfrage 2011

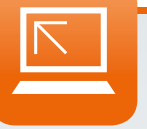

Top gelesen von Hausärzten auf springermedizin.de

- Trinker-Tod kommt selten von der Leber (2262828)

- Die sieben wichtigsten Empfehlungen für die COPD-Therapie (600770)

- Arzneinotfälle: meist sind Allerweltsmedikamente Schuld (2270634)

Loggen Sie sich ein und lesen Sie die Beiträge auf springermedizin.de! Die oben genannten Artikel finden Sie, indem Sie die ID-Nr. in die Suche eingeben. 